CRYSTALLOGRAPHIC COMMUNICATIONS

ISSN 2056-9890

Received 7 June 2015

Accepted 22 June 2015

Edited by W. T. A. Harrison, University of Aberdeen, Scotland

Keywords: crystal structure; chromone; hydrogen bonding; halogen interaction

CCDC reference: 1407902

Supporting information: this article has supporting information at journals.iucr.org/e

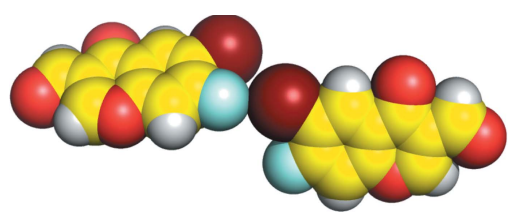

OPEN $\odot$ ACCESS

\section{Crystal structure of 6-bromo-7-fluoro-4-oxo-4H- chromene-3-carbaldehyde}

\author{
Yoshinobu Ishikawa
}

School of Pharmaceutical Sciences, University of Shizuoka, 52-1 Yada, Suruga-ku, Shizuoka 422-8526, Japan. *Correspondence e-mail: ishi206@u-shizuoka-ken.ac.jp

In the title compound, $\mathrm{C}_{10} \mathrm{H}_{4} \mathrm{BrFO}_{3}$, a brominated and fluorinated 3-formylchromone derivative, all atoms are essentially coplanar (r.m.s. deviation of $0.025 \AA$ for the non-H atoms), with the largest deviation from the least-squares plane $[0.050$ (6) $\AA$ ] being for a benzene-ring $C$ atom. In the crystal, molecules are linked through stacking interactions [centroid-centroid distance between the benzene and pyran rings $=3.912(4) \AA], C-H \cdots O$ hydrogen bonds and

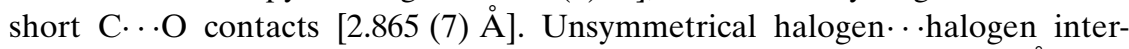
actions between the bromine and fluorine atoms $[\mathrm{Br} \cdots \mathrm{F}=3.116$ (4) $\AA$, C$\left.\mathrm{Br} \cdots \mathrm{F}=151.8(2), \mathrm{C}-\mathrm{F} \cdots \mathrm{Br}=154.1(4)^{\circ}\right]$ are also formed, giving a meandering two-dimensional network propagating in the (041) plane. A comparison with related structures is made and the various types of weak interactions are ranked in importance.

\section{Chemical context}

Halogen bonds and halogen...halogen interactions have recently attracted much attention in medicinal chemistry, chemical biology, supramolecular chemistry and crystal engineering (Auffinger et al., 2004; Metrangolo et al., 2005; Wilcken et al., 2013; Mukherjee \& Desiraju, 2014; Metrangolo \& Resnati, 2014; Persch et al., 2015). I have recently reported the crystal structures of the halogenated 3-formylchromone derivatives 6-chloro-4-oxo-4H-chromene-3-carbaldehyde (Ishikawa, 2014a), 6-bromo-4-oxo-4H-chromene-3-carbaldehyde (Ishikawa, 2014b) and 6-chloro-7-fluoro-4-oxo-4H-chromene3-carbaldehyde (Ishikawa, 2014c). A van der Waals contact between the formyl oxygen atom and the chlorine atom in 6chloro-4-oxo-4H-chromene-3-carbaldehyde (Fig. 1a) and a shorter contact (halogen bonding) between the formyl oxygen atom and the bromine atom in 6-bromo-4-oxo-4H-chromene3-carbaldehyde (Fig. 1b) are observed. On the other hand, an unsymmetrical halogen..halogen interaction is formed between the chlorine and fluorine atoms in 6-chloro-7-fluoro4-oxo-4H-chromene-3-carbaldehyde (Fig. 1c). As part of our interest in these types of chemical bonding, I herein report the crystal structure of a brominated and fluorinated 3-formylchromone derivative 6-bromo-7-fluoro-4-oxo-4H-chromene3 -carbaldehyde. The objective of this study is to reveal the inductive effect of the vicinal electron-withdrawing substituent on the bromine atom at the 6-position and the interaction $\operatorname{mode}(s)$.<smiles>O=Cc1coc2cc(F)c(Br)cc2c1=O</smiles> 

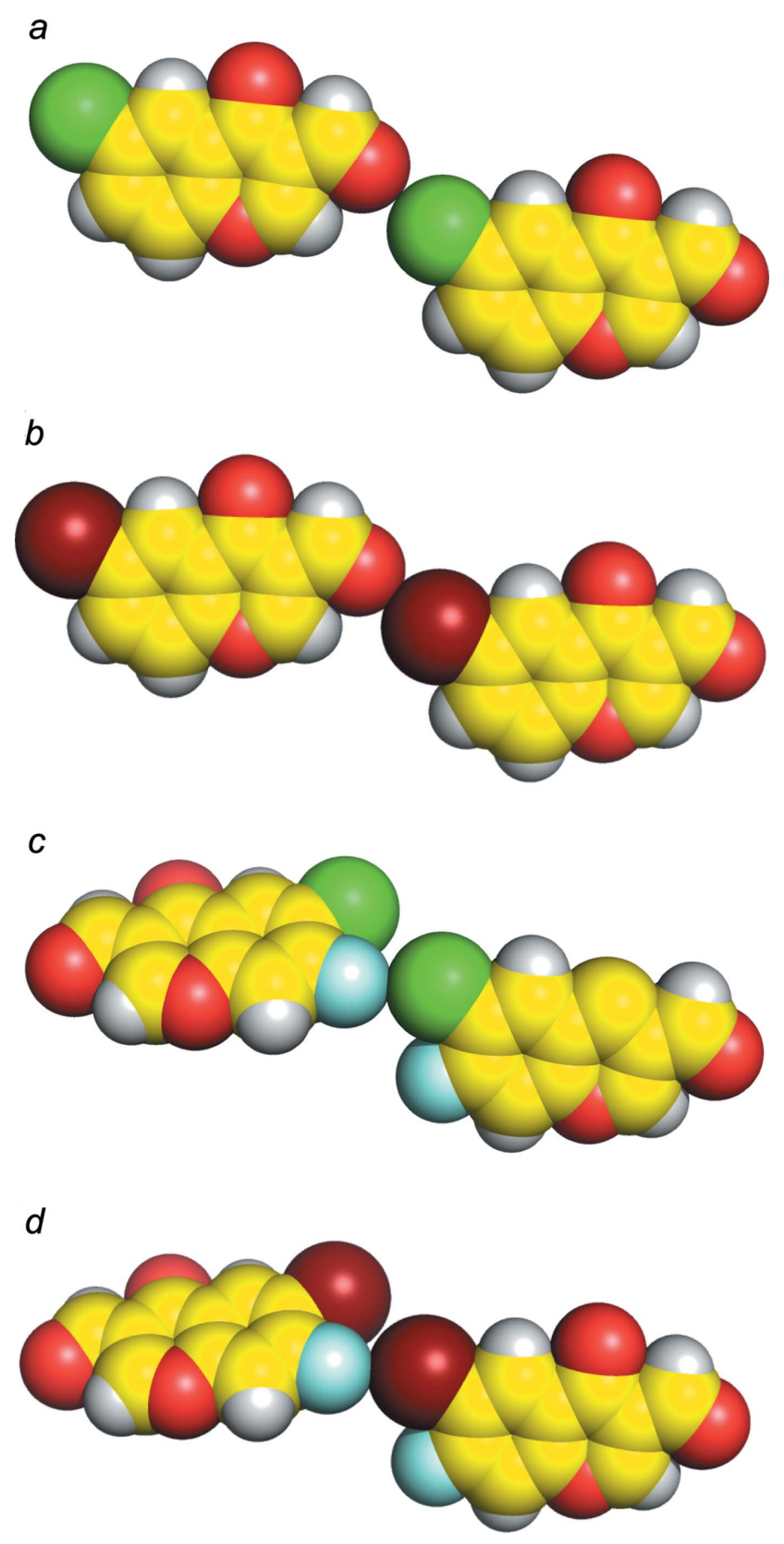

Figure 1

Sphere models of the crystal structures of (a) 6-chloro-4-oxo- $4 \mathrm{H}$ chromene-3-carbaldehyde (Ishikawa, 2014a), (b) 6-bromo-4-oxo-4Hchromene-3-carbaldehyde (Ishikawa, 2014b), (c) 6-chloro-7-fluoro-4oxo-4H-chromene-3-carbaldehyde (Ishikawa, 2014c) and $(d)$ the title compound.

\section{Structural commentary}

The title compound is shown in Fig. 2. The mean deviation of the least-square plane for the non-hydrogen atoms is $0.0253 \AA$, and the largest deviation is 0.050 (6) $\AA$ for C4. This means that these atoms are essentially coplanar.

\section{Supramolecular features}

In the crystal, the molecules are linked through stacking interactions between the translation-symmetry equivalent ${ }^{i}$

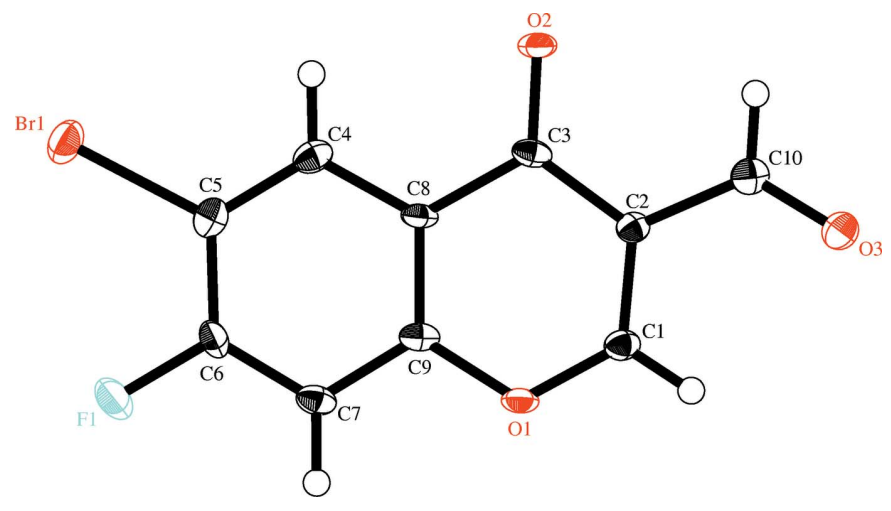

Figure 2

The molecular structure of the title compound, with displacement ellipsoids drawn at the $50 \%$ probability level. Hydrogen atoms are shown as small spheres of arbitrary radius.

[centroid-centroid distance between the benzene and pyran rings of the $4 H$-chromene units $=3.872$ (4) $\AA$, symmetry code: (i) $x, y, z-1]$, and through $\mathrm{C}-\mathrm{H} \cdots \mathrm{O}$ hydrogen bonds (Table 1), as shown in Fig. 3.

A contact between the formyl oxygen atom and the bromine atom is not found in the title compound. Instead, an unsymmetrical halogen..halogen interaction is formed between the bromine and fluorine atoms $[\mathrm{Br} 1 \cdots \mathrm{F} 1=$

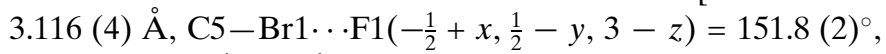
$\mathrm{C} 6-\mathrm{F} 1 \cdots \operatorname{Br} 1\left(\frac{1}{2}+x, \frac{1}{2}-y, 3-\mathrm{z}=154.1(4)^{\circ}\right]$, as shown in Fig. $1 d$. It is suggested that the electron-withdrawing fluorine atom at the 7-position should make the $\sigma$-hole of the bromine atom at the 6-position larger, and the electropositive region of the bromine atom should contact the electronegative region of the fluorine atom (Hathwar \& Guru Row, 2011). Thus, halogen bonds $(\mathrm{Cl} \cdots \mathrm{O}$ and $\mathrm{Br} \cdots \mathrm{O})$ are not observed in 6chloro-7-fluoro-4-oxo-4H-chromene-3-carbaldehyde and the title compound, which might support the idea that the unsymmetrical halogen $\cdots$ halogen interactions $(\mathrm{Cl} \cdots \mathrm{F}$ and $\mathrm{Br} \cdots \mathrm{F})$ are more favorable than the halogen bonds.

In addition to the $\mathrm{C}-\mathrm{H} \cdots \mathrm{O}$ hydrogen bonds and the unsymmetrical halogen . .halogen interaction, a short contact between the formyl $\mathrm{C} 10$ and $\mathrm{O} 3{ }^{\mathrm{ii}}$ atoms $\left[2.865\right.$ (7) $\AA$, (ii): $-x+\frac{1}{2}$, $-y, z+\frac{1}{2}$, Fig. 3] is revealed in the title compound. This extraordinary interaction is also observed in 6-chloro-7fluoro-4-oxo-4H-chromene-3-carbaldehyde (Ishikawa, 2014c), but is not observed in 6-chloro-4-oxo-4H-chromene-3-carbaldehyde (Ishikawa, 2014a), 6-bromo-4-oxo-4H-chromene-3carbaldehyde (Ishikawa 2014b) and 7-fluoro-4-oxochromene3-carbaldehyde (Asad et al., 2011). Thus, this interesting feature might be caused by a strong dipole-dipole interaction between the formyl groups polarized extremely by introducing

Table 1

Hydrogen-bond geometry $\left(\AA{ }^{\circ}\right)$.

\begin{tabular}{lllll}
\hline$D-\mathrm{H} \cdots A$ & $D-\mathrm{H}$ & $\mathrm{H} \cdots A$ & $D \cdots A$ & $D-\mathrm{H} \cdots A$ \\
\hline $\mathrm{C} 1-\mathrm{H} 1 \cdots \mathrm{O} 3^{\mathrm{i}}$ & 0.95 & 2.41 & $3.240(7)$ & 146 \\
$\mathrm{C} 7-\mathrm{H} 3 \cdots \mathrm{O} 2^{\mathrm{ii}}$ & 0.95 & 2.26 & $3.166(7)$ & 158 \\
\hline
\end{tabular}

Symmetry codes: (i) $-x+\frac{3}{2},-y, z+\frac{1}{2}$; (ii) $x+1, y, z+1$. 


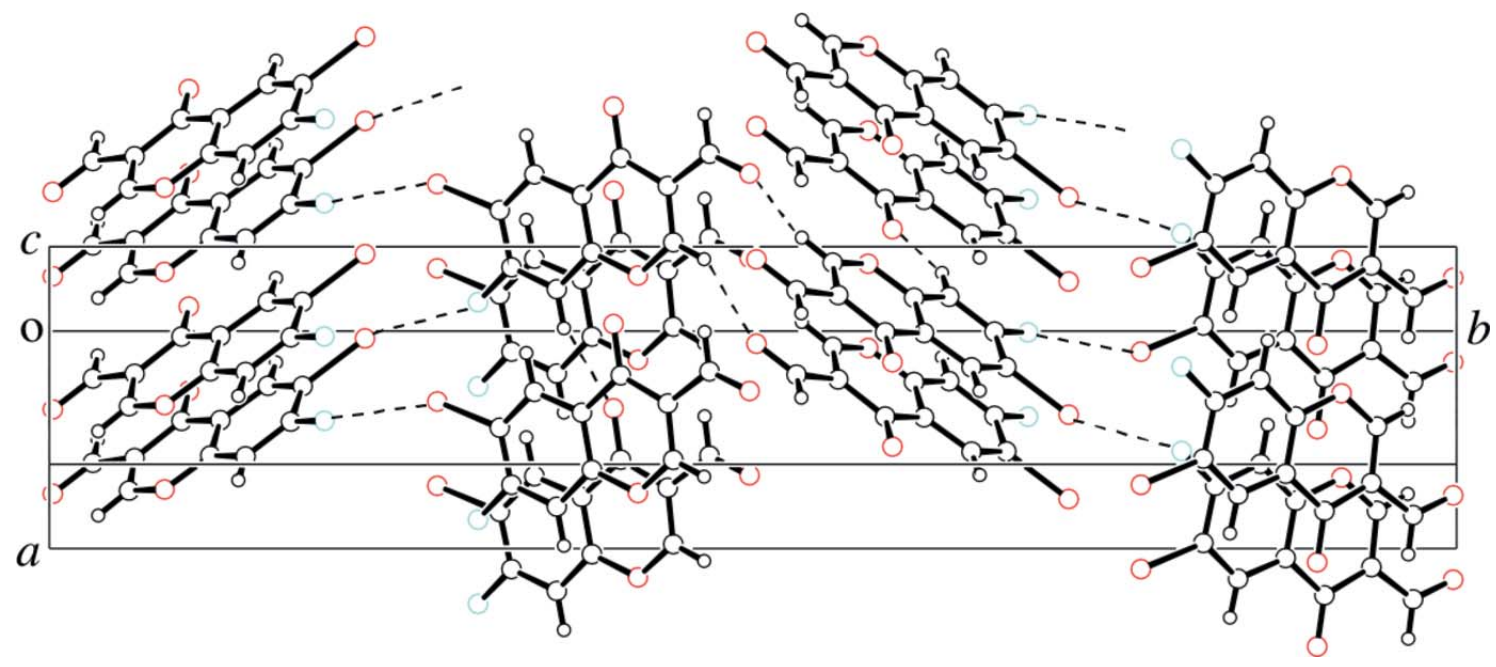

Figure 3

A packing view of the title compound. $\mathrm{C}-\mathrm{H} \cdots \mathrm{O}$ hydrogen bonds and $\mathrm{Br} \cdots \mathrm{F}$ unsymmetrical halogen $\cdots$ halogen interactions are represented as dashed lines.

both the bromine and fluorine atoms into the chromone ring. These findings should be helpful in the understanding of interactions of halogenated ligands with proteins, and thus invaluable for rational drug design.

\section{Synthesis and crystallization}

5-Bromo-4-fluoro-2-hydroxyacetophenone was prepared from 4-bromo-3-fluorophenol by Fries rearrangement reaction.

Table 2

Experimental details.

\begin{tabular}{|c|c|}
\hline \multicolumn{2}{|l|}{ Crystal data } \\
\hline Chemical formula & $\mathrm{C}_{10} \mathrm{H}_{4} \mathrm{BrFO}_{3}$ \\
\hline$M_{\mathrm{r}}$ & 271.04 \\
\hline Crystal system, space group & Orthorhombic, $P 2_{1} 2_{1} 2_{1}$ \\
\hline Temperature (K) & 100 \\
\hline$a, b, c(\AA)$ & $5.784(3), 33.713(14), 4.633(3)$ \\
\hline$V\left(\AA^{3}\right)$ & $903.4(8)$ \\
\hline$Z$ & 4 \\
\hline Radiation type & Mo $K \alpha$ \\
\hline$\mu\left(\mathrm{mm}^{-1}\right)$ & 4.56 \\
\hline Crystal size $(\mathrm{mm})$ & $0.30 \times 0.25 \times 0.10$ \\
\hline \multicolumn{2}{|l|}{ Data collection } \\
\hline Diffractometer & Rigaku AFC7R diffractometer \\
\hline Absorption correction & $\psi$ scan (North et al., 1968) \\
\hline$T_{\min }, T_{\max }$ & $0.388,0.634$ \\
\hline $\begin{array}{l}\text { No. of measured, independent and } \\
\text { observed }\left[F^{2}>2.0 \sigma\left(F^{2}\right)\right] \text { reflec- } \\
\text { tions }\end{array}$ & $1744,1384,1203$ \\
\hline$R_{\text {int }}$ & 0.033 \\
\hline$(\sin \theta / \lambda)_{\max }\left(\AA^{-1}\right)$ & 0.650 \\
\hline \multicolumn{2}{|l|}{ Refinement } \\
\hline$R\left[F^{2}>2 \sigma\left(F^{2}\right)\right], w R\left(F^{2}\right), S$ & $0.046,0.123,1.12$ \\
\hline No. of reflections & 1384 \\
\hline No. of parameters & 136 \\
\hline $\mathrm{H}$-atom treatment & $\mathrm{H}$-atom parameters constrained \\
\hline$\Delta \rho_{\max }, \Delta \rho_{\min }\left(\mathrm{e} \AA^{-3}\right)$ & $1.21,-1.53$ \\
\hline Absolute structure & Flack (1983), 110 Friedel pairs \\
\hline Absolute structure parameter & $0.02(3)$ \\
\hline
\end{tabular}

Computer programs: WinAFC (Rigaku, 1999), SIR2008 (Burla et al., 2007), SHELXL97 (Sheldrick, 2008), CrystalStructure (Rigaku, 2010).
To a solution of 5-bromo-4-fluoro-2-hydroxyacetophenone (7.56 mmol) in $N, N$-dimethylformamide $(15 \mathrm{ml})$ was added dropwise $\mathrm{POCl}_{3}(18.9 \mathrm{mmol})$ at $273 \mathrm{~K}$. After the mixture had been stirred for $14 \mathrm{~h}$ at room temperature, water $(50 \mathrm{ml})$ was added. The precipitates were collected, washed with water, and dried in vacuo (yield: $74 \%) .{ }^{1} \mathrm{H} \mathrm{NMR} \mathrm{(400} \mathrm{MHz}, \mathrm{CDCl}_{3}$ ): $\delta=7.33(d, 1 \mathrm{H}, J=8.0 \mathrm{~Hz}), 8.52(s, 1 \mathrm{H}), 8.54(s, 1 \mathrm{H}), 10.36(s$, $1 \mathrm{H})$. Colorless plates were obtained by slow evaporation of a 1,2-dimethoxyethane $/ n$-hexane solution of the title compound at room temperature.

\section{Refinement}

Crystal data, data collection and structure refinement details are summarized in Table 2. The Csp ${ }^{2}$-bound hydrogen atoms were placed in geometrical positions $\left[\mathrm{C}-\mathrm{H} 0.95 \AA, U_{\text {iso }}(\mathrm{H})=\right.$ $\left.1.2 U_{\text {eq }}(\mathrm{C})\right]$, and refined using a riding model.

\section{Acknowledgements}

I acknowledge University of Shizuoka for instrumental support.

\section{References}

Asad, M., Oo, C.-W., Osman, H., Hemamalini, M. \& Fun, H.-K. (2011). Acta Cryst. E67, o766.

Auffinger, P., Hays, F. A., Westhof, E. \& Ho, P. S. (2004). Proc. Natl Acad. Sci. USA, 101, 16789-16794.

Burla, M. C., Caliandro, R., Camalli, M., Carrozzini, B., Cascarano, G. L., De Caro, L., Giacovazzo, C., Polidori, G., Siliqi, D. \& Spagna, R. (2007). J. Appl. Cryst. 40, 609-613.

Flack, H. D. (1983). Acta Cryst. A39, 876-881.

Hathwar, V. R. \& Guru Row, T. N. (2011). Cryst. Growth Des. 11, $1338-1346$.

Ishikawa, Y. (2014a). Acta Cryst. E70, o514.

Ishikawa, Y. (2014b). Acta Cryst. E70, o555.

Ishikawa, Y. (2014c). Acta Cryst. E70, o825.

Metrangolo, P., Neukirch, H., Pilati, T. \& Resnati, G. (2005). Acc. Chem. Res. 38, 386-395. 
Metrangolo, P. \& Resnati, G. (2014). IUCrJ, 1, 5-7.

Mukherjee, A. \& Desiraju, G. R. (2014). IUCrJ, 1, 49-60.

North, A. C. T., Phillips, D. C. \& Mathews, F. S. (1968). Acta Cryst. A24, 351-359.

Persch, E., Dumele, O. \& Diederich, F. (2015). Angew. Chem. Int. Ed. 54, 3290-3327.
Rigaku (1999). WinAFC Diffractometer Control Software. Rigaku Corporation, Tokyo, Japan.

Rigaku (2010). CrystalStructure. Rigaku Corporation, Tokyo, Japan. Sheldrick, G. M. (2008). Acta Cryst. A64, 112-122.

Wilcken, R., Zimmermann, M. O., Lange, A., Joerger, A. C. \& Boeckler, F. M. (2013). J. Med. Chem. 56, 1363-1388. 


\section{supporting information}

Acta Cryst. (2015). E71, 857-860 [doi:10.1107/S2056989015011871]

\section{Crystal structure of 6-bromo-7-fluoro-4-oxo-4H-chromene-3-carbaldehyde}

\section{Yoshinobu Ishikawa}

\section{Computing details}

Data collection: WinAFC (Rigaku, 1999); cell refinement: WinAFC (Rigaku, 1999); data reduction: WinAFC (Rigaku, 1999); program(s) used to solve structure: SIR2008 (Burla et al., 2007); program(s) used to refine structure: SHELXL97 (Sheldrick, 2008); molecular graphics: CrystalStructure (Rigaku, 2010); software used to prepare material for publication: CrystalStructure (Rigaku, 2010).

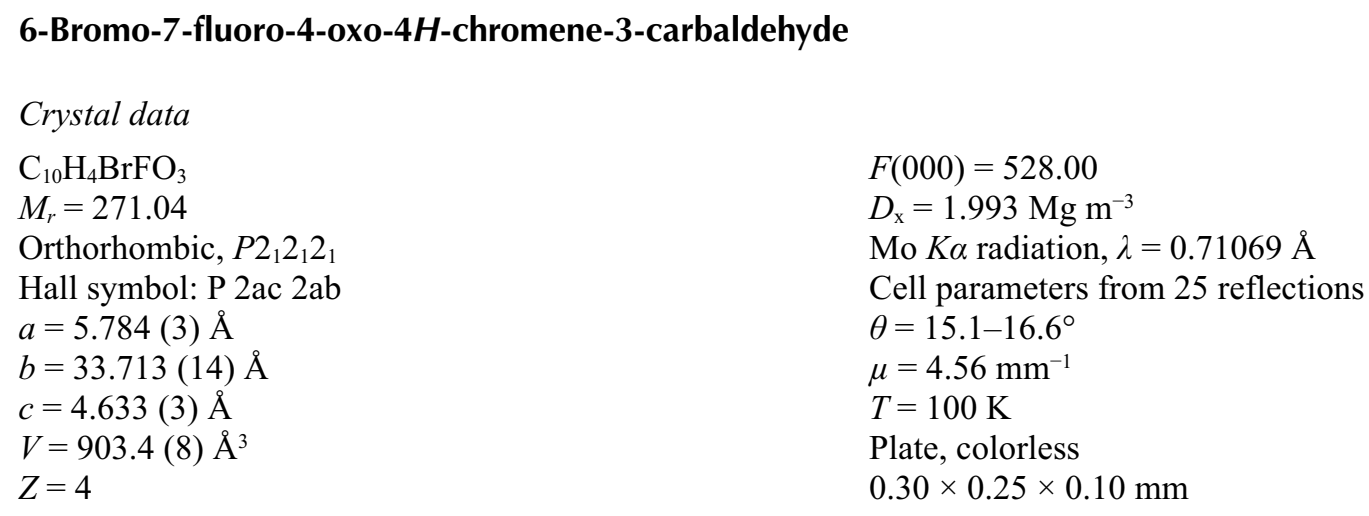

Data collection

Rigaku AFC7R

diffractometer

$\omega$ scans

Absorption correction: $\psi$ scan

(North et al., 1968)

$T_{\min }=0.388, T_{\max }=0.634$

1744 measured reflections

1384 independent reflections

Refinement

Refinement on $F^{2}$

$R\left[F^{2}>2 \sigma\left(F^{2}\right)\right]=0.046$

$w R\left(F^{2}\right)=0.123$

$S=1.12$

1384 reflections

136 parameters

0 restraints

Primary atom site location: structure-invariant direct methods

Secondary atom site location: difference Fourier map
1203 reflections with $F^{2}>2.0 \sigma\left(F^{2}\right)$

$R_{\text {int }}=0.033$

$\theta_{\max }=27.5^{\circ}$

$h=-7 \rightarrow 4$

$k=0 \rightarrow 43$

$l=-3 \rightarrow 6$

3 standard reflections every 150 reflections intensity decay: $-3.0 \%$

Hydrogen site location: inferred from neighbouring sites

$\mathrm{H}$-atom parameters constrained

$w=1 /\left[\sigma^{2}\left(F_{\mathrm{o}}^{2}\right)+(0.0881 P)^{2}\right]$

where $P=\left(F_{\mathrm{o}}{ }^{2}+2 F_{\mathrm{c}}{ }^{2}\right) / 3$

$(\Delta / \sigma)_{\max }<0.001$

$\Delta \rho_{\max }=1.21 \mathrm{e} \AA^{-3}$

$\Delta \rho_{\min }=-1.53$ e $\AA^{-3}$

Absolute structure: Flack (1983), 110 Friedel

Pairs

Absolute structure parameter: 0.02 (3) 
Special details

Refinement. Refinement was performed using all reflections. The weighted $R$-factor $(w R)$ and goodness of fit $(S)$ are based on $F^{2}$. $R$-factor (gt) are based on $F$. The threshold expression of $F^{2}>2.0 \sigma\left(F^{2}\right)$ is used only for calculating $R$-factor (gt).

Fractional atomic coordinates and isotropic or equivalent isotropic displacement parameters $\left(\AA^{2}\right)$

\begin{tabular}{lllll}
\hline & $x$ & $y$ & $z$ & $U_{\text {iso }} / U_{\text {eq }}$ \\
\hline Br1 & $0.12276(11)$ & $0.224324(15)$ & $1.22910(13)$ & $0.0241(2)$ \\
F1 & $0.5835(7)$ & $0.19539(10)$ & $1.4414(8)$ & $0.0246(8)$ \\
O1 & $0.6802(7)$ & $0.08180(12)$ & $0.8763(9)$ & $0.0163(9)$ \\
O2 & $0.0653(7)$ & $0.09910(11)$ & $0.4588(9)$ & $0.0181(9)$ \\
O3 & $0.4556(7)$ & $0.00248(11)$ & $0.2458(10)$ & $0.0206(9)$ \\
C1 & $0.6066(10)$ & $0.05663(16)$ & $0.6730(12)$ & $0.0156(11)$ \\
C2 & $0.4022(10)$ & $0.06057(15)$ & $0.5311(12)$ & $0.0136(11)$ \\
C3 & $0.2522(10)$ & $0.09394(16)$ & $0.5850(12)$ & $0.0146(12)$ \\
C4 & $0.2095(10)$ & $0.15532(16)$ & $0.8912(13)$ & $0.0174(12)$ \\
C5 & $0.2924(11)$ & $0.17998(16)$ & $1.1017(14)$ & $0.0174(12)$ \\
C6 & $0.5054(10)$ & $0.17161(15)$ & $1.2299(14)$ & $0.0174(11)$ \\
C7 & $0.6356(10)$ & $0.13944(16)$ & $1.1538(13)$ & $0.0166(11)$ \\
C8 & $0.3361(9)$ & $0.12153(16)$ & $0.8105(12)$ & $0.0143(12)$ \\
C9 & $0.5490(10)$ & $0.11445(16)$ & $0.9405(12)$ & $0.0142(12)$ \\
C10 & $0.3392(10)$ & $0.03094(16)$ & $0.3123(12)$ & $0.0173(12)$ \\
H1 & 0.7026 & 0.0347 & 0.6250 & $0.0187^{*}$ \\
H2 & 0.0664 & 0.1611 & 0.7995 & $0.0209^{*}$ \\
H3 & 0.7803 & 0.1343 & 1.2435 & $0.0199 *$ \\
H4 & 0.1959 & 0.0344 & 0.2153 & $0.0208^{*}$ \\
& & & & \\
\hline
\end{tabular}

Atomic displacement parameters $\left(\AA^{2}\right)$

\begin{tabular}{lllllll}
\hline & $U^{11}$ & $U^{22}$ & $U^{33}$ & $U^{12}$ & $U^{13}$ & $U^{23}$ \\
\hline Br1 & $0.0215(4)$ & $0.0219(3)$ & $0.0289(4)$ & $0.0043(3)$ & $0.0054(3)$ & $-0.0050(3)$ \\
F1 & $0.0229(19)$ & $0.0293(18)$ & $0.0217(17)$ & $-0.0055(16)$ & $-0.0022(18)$ & $-0.0089(15)$ \\
O1 & $0.0088(19)$ & $0.024(2)$ & $0.0159(19)$ & $0.0017(16)$ & $-0.0026(17)$ & $-0.0016(17)$ \\
O2 & $0.012(2)$ & $0.024(2)$ & $0.018(2)$ & $0.0042(17)$ & $-0.0052(18)$ & $-0.0009(17)$ \\
O3 & $0.0170(19)$ & $0.0243(19)$ & $0.020(2)$ & $0.0020(16)$ & $0.002(2)$ & $-0.0038(19)$ \\
C1 & $0.012(3)$ & $0.021(3)$ & $0.014(3)$ & $0.002(3)$ & $0.001(3)$ & $0.000(2)$ \\
C2 & $0.014(3)$ & $0.016(3)$ & $0.011(3)$ & $0.002(3)$ & $0.000(3)$ & $0.0019(19)$ \\
C3 & $0.010(3)$ & $0.022(3)$ & $0.012(3)$ & $-0.003(3)$ & $0.000(3)$ & $0.003(3)$ \\
C4 & $0.014(3)$ & $0.021(3)$ & $0.018(3)$ & $0.003(3)$ & $0.002(3)$ & $0.003(3)$ \\
C5 & $0.019(3)$ & $0.015(3)$ & $0.018(3)$ & $0.000(3)$ & $0.005(3)$ & $0.001(3)$ \\
C6 & $0.020(3)$ & $0.016(3)$ & $0.016(3)$ & $-0.003(3)$ & $-0.001(3)$ & $-0.005(3)$ \\
C7 & $0.011(3)$ & $0.024(3)$ & $0.015(3)$ & $-0.003(3)$ & $-0.001(3)$ & $0.001(2)$ \\
C8 & $0.009(3)$ & $0.023(3)$ & $0.011(3)$ & $0.001(2)$ & $-0.002(3)$ & $0.001(2)$ \\
C9 & $0.010(3)$ & $0.023(3)$ & $0.010(3)$ & $-0.001(2)$ & $0.001(2)$ & $0.005(3)$ \\
C10 & $0.016(3)$ & $0.023(3)$ & $0.013(3)$ & $0.001(3)$ & $0.004(3)$ & $0.002(3)$ \\
& & & & & &
\end{tabular}


Geometric parameters $\left(\AA,{ }^{\circ}\right)$

\begin{tabular}{|c|c|c|c|}
\hline $\mathrm{Br} 1-\mathrm{C} 5$ & $1.883(6)$ & $\mathrm{C} 4-\mathrm{C} 5$ & $1.369(9)$ \\
\hline $\mathrm{F} 1-\mathrm{C} 6$ & $1.344(7)$ & $\mathrm{C} 4-\mathrm{C} 8$ & $1.405(8)$ \\
\hline $\mathrm{O} 1-\mathrm{C} 1$ & $1.338(7)$ & $\mathrm{C} 5-\mathrm{C} 6$ & $1.396(9)$ \\
\hline $\mathrm{O} 1-\mathrm{C} 9$ & $1.370(7)$ & $\mathrm{C} 6-\mathrm{C} 7$ & $1.367(8)$ \\
\hline $\mathrm{O} 2-\mathrm{C} 3$ & $1.241(7)$ & $\mathrm{C} 7-\mathrm{C} 9$ & $1.392(8)$ \\
\hline $\mathrm{O} 3-\mathrm{C} 10$ & $1.212(7)$ & $\mathrm{C} 8-\mathrm{C} 9$ & $1.392(8)$ \\
\hline $\mathrm{C} 1-\mathrm{C} 2$ & $1.359(8)$ & $\mathrm{C} 1-\mathrm{H} 1$ & 0.950 \\
\hline $\mathrm{C} 2-\mathrm{C} 3$ & $1.443(8)$ & $\mathrm{C} 4-\mathrm{H} 2$ & 0.950 \\
\hline $\mathrm{C} 2-\mathrm{C} 10$ & $1.469(8)$ & $\mathrm{C} 7-\mathrm{H} 3$ & 0.950 \\
\hline $\mathrm{C} 3-\mathrm{C} 8$ & $1.480(8)$ & $\mathrm{C} 10-\mathrm{H} 4$ & 0.950 \\
\hline $\mathrm{Br} 1 \cdots \mathrm{F} 1$ & $3.004(4)$ & $\mathrm{Br} 1 \cdots \mathrm{H} 2$ & 2.9352 \\
\hline $\mathrm{F} 1 \cdots \mathrm{C} 9$ & $3.588(7)$ & $\mathrm{F} 1 \cdots \mathrm{H} 3$ & 2.5252 \\
\hline $\mathrm{O} 1 \cdots \mathrm{C} 3$ & $2.849(7)$ & $\mathrm{O} 1 \cdots \mathrm{H} 3$ & 2.5222 \\
\hline $\mathrm{O} 1 \cdots \mathrm{C} 6$ & $3.588(7)$ & $\mathrm{O} 2 \cdots \mathrm{H} 2$ & 2.6181 \\
\hline $\mathrm{O} 2 \cdots \mathrm{C} 1$ & $3.583(7)$ & $\mathrm{O} 2 \cdots \mathrm{H} 4$ & 2.5704 \\
\hline $\mathrm{O} 2 \cdots \mathrm{C} 4$ & $2.881(7)$ & O3 $\cdots \mathrm{H} 1$ & 2.5121 \\
\hline $\mathrm{O} 2 \cdots \mathrm{C} 10$ & $2.873(7)$ & $\mathrm{C} 1 \cdots \mathrm{H} 4$ & 3.2714 \\
\hline $\mathrm{O} 3 \cdots \mathrm{C} 1$ & $2.831(7)$ & $\mathrm{C} 3 \cdots \mathrm{H} 1$ & 3.2873 \\
\hline $\mathrm{C} 1 \cdots \mathrm{C} 7$ & $3.576(8)$ & $\mathrm{C} 3 \cdots \mathrm{H} 2$ & 2.6949 \\
\hline $\mathrm{C} 1 \cdots \mathrm{C} 8$ & $2.764(8)$ & $\mathrm{C} 3 \cdots \mathrm{H} 4$ & 2.6597 \\
\hline $\mathrm{C} 2 \cdots \mathrm{C} 9$ & $2.760(8)$ & $\mathrm{C} 5 \cdots \mathrm{H} 3$ & 3.2809 \\
\hline $\mathrm{C} 4 \cdots \mathrm{C} 7$ & $2.800(8)$ & $\mathrm{C} 6 \cdots \mathrm{H} 2$ & 3.2480 \\
\hline $\mathrm{C} 5 \cdots \mathrm{C} 9$ & $2.764(8)$ & $\mathrm{C} 8 \cdots \mathrm{H} 3$ & 3.2878 \\
\hline $\mathrm{C} 6 \cdots \mathrm{C} 8$ & $2.754(8)$ & $\mathrm{C} 9 \cdots \mathrm{H} 1$ & 3.1856 \\
\hline $\mathrm{Br} 1 \cdots \mathrm{F} 1^{\mathrm{i}}$ & $3.413(4)$ & $\mathrm{C} 9 \cdots \mathrm{H} 2$ & 3.2692 \\
\hline $\mathrm{Br} 1 \cdots \mathrm{F} 1^{\mathrm{ii}}$ & $3.116(4)$ & $\mathrm{C} 10 \cdots \mathrm{H} 1$ & 2.5561 \\
\hline $\mathrm{F} 1 \cdots \mathrm{Br} 1^{\mathrm{iii}}$ & $3.413(4)$ & $\mathrm{H} 1 \cdots \mathrm{H} 4$ & 3.4920 \\
\hline $\mathrm{F} 1 \cdots \mathrm{Br} 1^{\text {iv }}$ & $3.116(4)$ & $\mathrm{Br} 1 \cdots \mathrm{H} 2^{\mathrm{v}}$ & 3.4115 \\
\hline $\mathrm{F} 1 \cdots \mathrm{C} 4^{\mathrm{v}}$ & $3.293(7)$ & $\mathrm{F} 1 \cdots \mathrm{H} 2^{\mathrm{vi}}$ & 3.4488 \\
\hline $\mathrm{F} 1 \cdots \mathrm{C} 5^{\mathrm{v}}$ & $3.530(8)$ & $\mathrm{O} 1 \cdots \mathrm{H} 2^{\mathrm{iii}}$ & 3.5011 \\
\hline $\mathrm{F} 1 \cdots \mathrm{C} 8^{\mathrm{v}}$ & $3.342(7)$ & $\mathrm{O} 1 \cdots \mathrm{H} 3^{\text {ix }}$ & 3.4733 \\
\hline $\mathrm{F} 1 \cdots \mathrm{C} 9^{\mathrm{v}}$ & $3.582(7)$ & $\mathrm{O} 1 \cdots \mathrm{H} 4^{\mathrm{v}}$ & 3.5880 \\
\hline $\mathrm{O} 1 \cdots \mathrm{O} 2^{\mathrm{iii}}$ & $3.007(6)$ & $\mathrm{O} 2 \cdots \mathrm{H} 1^{\mathrm{i}}$ & 3.1145 \\
\hline $\mathrm{O} 1 \cdots \mathrm{O} 2^{\mathrm{vi}}$ & $3.547(6)$ & $\mathrm{O} 2 \cdots \mathrm{H} 3^{\text {viii }}$ & 2.2632 \\
\hline $\mathrm{O} 1 \cdots \mathrm{O}^{\mathrm{v}}$ & $3.431(6)$ & $\mathrm{O} 3 \cdots \mathrm{H} 1^{\mathrm{ix}}$ & 3.3905 \\
\hline $\mathrm{O} 1 \cdots \mathrm{O} 3^{\mathrm{vii}}$ & $3.589(6)$ & $\mathrm{O} 3 \cdots \mathrm{H} 1^{\mathrm{x}}$ & 2.4077 \\
\hline $\mathrm{O} 1 \cdots \mathrm{C} 2^{\mathrm{v}}$ & $3.507(7)$ & $\mathrm{O} 3 \cdots \mathrm{H} 4^{\mathrm{xi}}$ & 2.8893 \\
\hline $\mathrm{O} 1 \cdots \mathrm{C} 3^{\mathrm{iii}}$ & $3.596(7)$ & $\mathrm{O} 3 \cdots \mathrm{H} 4^{\mathrm{xii}}$ & 2.6535 \\
\hline $\mathrm{O} 1 \cdots \mathrm{C} 10^{\mathrm{v}}$ & $3.303(7)$ & $\mathrm{C} 1 \cdots \mathrm{H} 3^{\mathrm{ix}}$ & 3.4389 \\
\hline $\mathrm{O} 2 \cdots \mathrm{O} 1^{\mathrm{viii}}$ & $3.547(6)$ & $\mathrm{C} 1 \cdots \mathrm{H} 4^{\mathrm{v}}$ & 3.5384 \\
\hline $\mathrm{O} 2 \cdots \mathrm{O} 1^{\mathrm{i}}$ & $3.007(6)$ & $\mathrm{C} 1 \cdots \mathrm{H} 4^{\mathrm{xii}}$ & 3.5368 \\
\hline $\mathrm{O} 2 \cdots \mathrm{C} 1^{\mathrm{i}}$ & $3.174(7)$ & $\mathrm{C} 2 \cdots \mathrm{H} 3^{\mathrm{ix}}$ & 3.5689 \\
\hline $\mathrm{O} 2 \cdots \mathrm{C} 4^{\mathrm{ix}}$ & $3.347(8)$ & $\mathrm{C} 2 \cdots \mathrm{H} 4^{\mathrm{v}}$ & 3.5005 \\
\hline $\mathrm{O} 2 \cdots \mathrm{C}^{\mathrm{ix}}$ & $3.449(7)$ & $\mathrm{C} 2 \cdots \mathrm{H} 4^{\mathrm{xii}}$ & 3.3605 \\
\hline $\mathrm{O} 2 \cdots \mathrm{C} 7^{\text {viii }}$ & $3.166(7)$ & $\mathrm{C} 3 \cdots \mathrm{H} 3^{\text {viii }}$ & 3.4362 \\
\hline
\end{tabular}




\begin{tabular}{|c|c|c|c|}
\hline $\mathrm{O} 2 \cdots \mathrm{C} 8^{\mathrm{ix}}$ & $3.471(7)$ & $\mathrm{C} 3 \cdots \mathrm{H} 4^{\mathrm{v}}$ & 3.5589 \\
\hline $\mathrm{O} 3 \cdots \mathrm{O} 1^{\mathrm{ix}}$ & $3.431(6)$ & $\mathrm{C} 4 \cdots \mathrm{H} 3^{\mathrm{i}}$ & 3.0546 \\
\hline $\mathrm{O} 3 \cdots \mathrm{O} 1^{\mathrm{x}}$ & $3.589(6)$ & $\mathrm{C} 5 \cdots \mathrm{H} 2^{v}$ & 3.5448 \\
\hline $\mathrm{O} 3 \cdots \mathrm{O} 3^{\mathrm{xi}}$ & $3.324(6)$ & $\mathrm{C} 5 \cdots \mathrm{H} 3^{\mathrm{i}}$ & 3.4028 \\
\hline $\mathrm{O} 3 \cdots \mathrm{O} 3^{\mathrm{xii}}$ & $3.324(6)$ & $\mathrm{C} 7 \cdots \mathrm{H} 2^{\mathrm{iii}}$ & 3.0718 \\
\hline $\mathrm{O} 3 \cdots \mathrm{C} 1^{\text {ix }}$ & $3.337(7)$ & $\mathrm{C} 8 \cdots \mathrm{H} 4^{v}$ & 3.5793 \\
\hline $\mathrm{O} 3 \cdots \mathrm{C} 1^{\mathrm{x}}$ & $3.240(7)$ & $\mathrm{C} 9 \cdots \mathrm{H} 2^{\mathrm{iii}}$ & 3.4429 \\
\hline $\mathrm{O} 3 \cdots \mathrm{C} 2^{\mathrm{xi}}$ & $3.129(7)$ & $\mathrm{C} 9 \cdots \mathrm{H} 3^{\text {ix }}$ & 3.5587 \\
\hline $\mathrm{O} 3 \cdots \mathrm{C} 3^{\mathrm{xi}}$ & $3.545(7)$ & $\mathrm{C} 10 \cdots \mathrm{H} 1^{\mathrm{x}}$ & 3.5611 \\
\hline $\mathrm{O} 3 \cdots \mathrm{C} 10^{\mathrm{xi}}$ & $2.865(7)$ & $\mathrm{C} 10 \cdots \mathrm{H} 4^{\mathrm{xi}}$ & 3.5406 \\
\hline $\mathrm{O} 3 \cdots \mathrm{C} 10^{\mathrm{xii}}$ & $3.326(7)$ & $\mathrm{C} 10 \cdots \mathrm{H} 4^{\mathrm{xii}}$ & 2.8936 \\
\hline $\mathrm{C} 1 \cdots \mathrm{O} 2^{\mathrm{iii}}$ & $3.174(7)$ & $\mathrm{H} 1 \cdots \mathrm{O} 2^{\mathrm{iii}}$ & 3.1145 \\
\hline $\mathrm{C} 1 \cdots \mathrm{O}^{\mathrm{v}}$ & $3.337(7)$ & $\mathrm{H} 1 \cdots \mathrm{O}^{\mathrm{v}}$ & 3.3905 \\
\hline $\mathrm{C} 1 \cdots \mathrm{O} 3^{\mathrm{vii}}$ & $3.240(7)$ & $\mathrm{H} 1 \cdots \mathrm{O} 3^{\text {vii }}$ & 2.4077 \\
\hline $\mathrm{C} 1 \cdots \mathrm{C} 10^{\mathrm{v}}$ & $3.452(8)$ & $\mathrm{H} 1 \cdots \mathrm{C} 10^{\mathrm{vii}}$ & 3.5611 \\
\hline $\mathrm{C} 2 \cdots \mathrm{O} 1^{\mathrm{ix}}$ & $3.507(7)$ & $\mathrm{H} 1 \cdots \mathrm{H} 1^{\mathrm{x}}$ & 3.3395 \\
\hline $\mathrm{C} 2 \cdots \mathrm{O} 3^{\mathrm{xii}}$ & $3.129(7)$ & $\mathrm{H} 1 \cdots \mathrm{H} 1^{\mathrm{vii}}$ & 3.3395 \\
\hline$C 2 \cdots C 7^{\text {ix }}$ & $3.456(8)$ & $\mathrm{H} 1 \cdots \mathrm{H} 4^{\mathrm{iii}}$ & 3.4266 \\
\hline$C 2 \cdots C 9^{\text {ix }}$ & $3.392(8)$ & $\mathrm{H} 1 \cdots \mathrm{H} 4^{\mathrm{xii}}$ & 3.3038 \\
\hline $\mathrm{C} 3 \cdots \mathrm{O} 1^{\mathrm{i}}$ & $3.596(7)$ & $\mathrm{H} 2 \cdots \mathrm{Br} 1^{\mathrm{ix}}$ & 3.4115 \\
\hline $\mathrm{C} 3 \cdots 3^{\mathrm{xii}}$ & $3.545(7)$ & $\mathrm{H} 2 \cdots \mathrm{F} 1^{\text {viii }}$ & 3.4488 \\
\hline $\mathrm{C} 3 \cdots \mathrm{C} 6^{\mathrm{ix}}$ & $3.422(8)$ & $\mathrm{H} 2 \cdots \mathrm{O} 1^{\mathrm{i}}$ & 3.5011 \\
\hline $\mathrm{C} 3 \cdots \mathrm{C}^{\mathrm{ix}}$ & $3.356(8)$ & $\mathrm{H} 2 \cdots \mathrm{C} 5^{\text {ix }}$ & 3.5448 \\
\hline$C 3 \cdots C 9^{\text {ix }}$ & $3.513(8)$ & $\mathrm{H} 2 \cdots \mathrm{C} 7^{\mathrm{i}}$ & 3.0718 \\
\hline $\mathrm{C} 4 \cdots \mathrm{F} 1^{\text {ix }}$ & $3.293(7)$ & $\mathrm{H} 2 \cdots \mathrm{C} 9^{\mathrm{i}}$ & 3.4429 \\
\hline $\mathrm{C} 4 \cdots \mathrm{O} 2^{\mathrm{v}}$ & $3.347(8)$ & $\mathrm{H} 2 \cdots \mathrm{H} 3^{\text {viii }}$ & 3.1920 \\
\hline$C 4 \cdots C 6^{\text {ix }}$ & $3.552(9)$ & $\mathrm{H} 2 \cdots \mathrm{H} 3^{\mathrm{i}}$ & 2.7898 \\
\hline $\mathrm{C} 4 \cdots \mathrm{C} 7^{\mathrm{i}}$ & $3.576(9)$ & $\mathrm{H} 3 \cdots \mathrm{O} 1^{\mathrm{v}}$ & 3.4733 \\
\hline $\mathrm{C} 5 \cdots \mathrm{F} 1^{\mathrm{ix}}$ & $3.530(8)$ & $\mathrm{H} 3 \cdots \mathrm{O} 2^{\mathrm{vi}}$ & 2.2632 \\
\hline $\mathrm{C} 5 \cdots \mathrm{O} 2^{\mathrm{v}}$ & $3.449(7)$ & $\mathrm{H} 3{ }^{\cdots} \mathrm{C} 1^{\mathrm{v}}$ & 3.4389 \\
\hline $\mathrm{C} 6 \cdots \mathrm{C}^{\mathrm{v}}$ & $3.422(8)$ & $\mathrm{H} 3{ }^{\cdots} \mathrm{C} 2^{\mathrm{v}}$ & 3.5689 \\
\hline $\mathrm{C} 6{ }^{\cdots} \mathrm{C} 4^{\mathrm{v}}$ & $3.552(9)$ & $\mathrm{H} 3{ }^{\cdots} \mathrm{C}^{\mathrm{vi}}$ & 3.4362 \\
\hline $\mathrm{C} 6 \cdots \mathrm{C}^{\mathrm{v}}$ & $3.323(9)$ & $\mathrm{H} 3 \cdots_{\mathrm{C}} 4^{\mathrm{iii}}$ & 3.0546 \\
\hline $\mathrm{C} 7 \cdots \mathrm{O} 2^{\mathrm{vi}}$ & $3.166(7)$ & $\mathrm{H} 3 \cdots \mathrm{C} 5^{\mathrm{iii}}$ & 3.4028 \\
\hline $\mathrm{C} 7 \cdots \mathrm{C} 2^{\mathrm{v}}$ & $3.456(8)$ & $\mathrm{H} 3{ }^{\cdots} \mathrm{C} 9^{v}$ & 3.5587 \\
\hline $\mathrm{C} 7 \cdots \mathrm{C}^{\mathrm{v}}$ & $3.356(8)$ & $\mathrm{H} 3 \cdots \mathrm{H} 2^{\mathrm{iii}}$ & 2.7898 \\
\hline $\mathrm{C} 7 \cdots \mathrm{C}^{\mathrm{iii}}$ & $3.576(9)$ & $\mathrm{H} 3{ }^{\cdots} \cdot \mathrm{H} 2^{\mathrm{vi}}$ & 3.1920 \\
\hline $\mathrm{C} 7 \cdots \mathrm{C} 8^{\mathrm{v}}$ & $3.552(8)$ & $\mathrm{H} 4 \cdots \mathrm{O} 1^{\text {ix }}$ & 3.5880 \\
\hline $\mathrm{C} 8 \cdots \mathrm{F} 1^{\text {ix }}$ & $3.342(7)$ & $\mathrm{H} 4 \cdots \mathrm{O} 3^{\mathrm{xi}}$ & 2.6535 \\
\hline $\mathrm{C} 8 \cdots \mathrm{O} 2^{v}$ & $3.471(7)$ & $\mathrm{H} 4 \cdots \mathrm{O}^{\mathrm{xii}}$ & 2.8893 \\
\hline$C 8 \cdots C 6^{\text {ix }}$ & $3.323(9)$ & $\mathrm{H} 4 \cdots \mathrm{C} 1^{\text {ix }}$ & 3.5384 \\
\hline$C 8 \cdots C 7^{\text {ix }}$ & $3.552(8)$ & $\mathrm{H} 4 \cdots \mathrm{C} 1^{\mathrm{xi}}$ & 3.5368 \\
\hline $\mathrm{C} 9 \cdots \mathrm{F} 1^{\mathrm{ix}}$ & $3.582(7)$ & $\mathrm{H} 4 \cdots \mathrm{C} 2^{\mathrm{ix}}$ & 3.5005 \\
\hline $\mathrm{C} 9 \cdots \mathrm{C} 2^{\mathrm{v}}$ & $3.392(8)$ & $\mathrm{H} 4 \cdots \mathrm{C} 2^{\mathrm{xi}}$ & 3.3605 \\
\hline $\mathrm{C} 9 \cdots \mathrm{C}^{\mathrm{v}}$ & $3.513(8)$ & $\mathrm{H} 4{ }^{\cdots} \mathrm{C} 3^{\text {ix }}$ & 3.5589 \\
\hline $\mathrm{C} 9 \cdots \mathrm{C} 10^{\mathrm{v}}$ & $3.517(8)$ & $\mathrm{H} 4 \cdots \mathrm{C}^{8 \mathrm{ix}}$ & 3.5793 \\
\hline $\mathrm{C} 10 \cdots \mathrm{O} 1^{\mathrm{ix}}$ & $3.303(7)$ & $\mathrm{H} 4{ }^{\cdots} \cdot \mathrm{C} 10^{\mathrm{xi}}$ & 2.8936 \\
\hline $\mathrm{C} 10 \cdots \mathrm{O} 3^{\mathrm{xi}}$ & $3.326(7)$ & $\mathrm{H} 4 \cdots \mathrm{C} 10^{\mathrm{xii}}$ & 3.5406 \\
\hline
\end{tabular}




\begin{tabular}{|c|c|c|c|}
\hline $\mathrm{C} 10 \cdots \mathrm{O}^{\mathrm{xii}}$ & $2.865(7)$ & $\mathrm{H} 4 \cdots \cdot \mathrm{H} 1^{\mathrm{i}}$ & 3.4266 \\
\hline $\mathrm{C} 10 \cdots \mathrm{C} 1^{\mathrm{ix}}$ & $3.452(8)$ & $\mathrm{H} 4 \cdots \mathrm{H} 1^{\mathrm{xi}}$ & 3.3038 \\
\hline $\mathrm{C} 10 \cdots \mathrm{C} 9^{\text {ix }}$ & $3.517(8)$ & $\mathrm{H} 4 \cdots \mathrm{H} 4^{\mathrm{xi}}$ & 3.3354 \\
\hline $\mathrm{C} 10 \cdots \mathrm{C} 10^{\mathrm{xi}}$ & $3.284(8)$ & $\mathrm{H} 4 \cdots \mathrm{H} 4^{\mathrm{xii}}$ & 3.3354 \\
\hline $\mathrm{C} 10 \cdots \mathrm{C} 10^{\mathrm{xii}}$ & $3.284(8)$ & & \\
\hline $\mathrm{C} 1-\mathrm{O} 1-\mathrm{C} 9$ & $119.1(5)$ & $\mathrm{C} 3-\mathrm{C} 8-\mathrm{C} 4$ & $121.8(5)$ \\
\hline $\mathrm{O} 1-\mathrm{C} 1-\mathrm{C} 2$ & $123.8(5)$ & $\mathrm{C} 3-\mathrm{C} 8-\mathrm{C} 9$ & $119.2(5)$ \\
\hline $\mathrm{C} 1-\mathrm{C} 2-\mathrm{C} 3$ & $121.0(5)$ & $\mathrm{C} 4-\mathrm{C} 8-\mathrm{C} 9$ & $119.0(5)$ \\
\hline $\mathrm{C} 1-\mathrm{C} 2-\mathrm{C} 10$ & $118.9(5)$ & $\mathrm{O} 1-\mathrm{C} 9-\mathrm{C} 7$ & $116.2(5)$ \\
\hline $\mathrm{C} 3-\mathrm{C} 2-\mathrm{C} 10$ & $120.0(5)$ & $\mathrm{O} 1-\mathrm{C} 9-\mathrm{C} 8$ & $122.3(5)$ \\
\hline $\mathrm{O} 2-\mathrm{C} 3-\mathrm{C} 2$ & $123.5(5)$ & $\mathrm{C} 7-\mathrm{C} 9-\mathrm{C} 8$ & $121.5(5)$ \\
\hline $\mathrm{O} 2-\mathrm{C} 3-\mathrm{C} 8$ & $122.0(5)$ & $\mathrm{O} 3-\mathrm{C} 10-\mathrm{C} 2$ & $125.2(6)$ \\
\hline $\mathrm{C} 2-\mathrm{C} 3-\mathrm{C} 8$ & $114.5(5)$ & $\mathrm{O} 1-\mathrm{C} 1-\mathrm{H} 1$ & 118.124 \\
\hline $\mathrm{C} 5-\mathrm{C} 4-\mathrm{C} 8$ & $120.0(6)$ & $\mathrm{C} 2-\mathrm{C} 1-\mathrm{H} 1$ & 118.120 \\
\hline $\mathrm{Br} 1-\mathrm{C} 5-\mathrm{C} 4$ & $121.5(5)$ & $\mathrm{C} 5-\mathrm{C} 4-\mathrm{H} 2$ & 120.003 \\
\hline $\mathrm{Br} 1-\mathrm{C} 5-\mathrm{C} 6$ & $119.1(5)$ & $\mathrm{C} 8-\mathrm{C} 4-\mathrm{H} 2$ & 120.030 \\
\hline $\mathrm{C} 4-\mathrm{C} 5-\mathrm{C} 6$ & $119.3(6)$ & $\mathrm{C} 6-\mathrm{C} 7-\mathrm{H} 3$ & 121.140 \\
\hline $\mathrm{F} 1-\mathrm{C} 6-\mathrm{C} 5$ & $119.1(5)$ & $\mathrm{C} 9-\mathrm{C} 7-\mathrm{H} 3$ & 121.139 \\
\hline $\mathrm{F} 1-\mathrm{C} 6-\mathrm{C} 7$ & $118.4(5)$ & $\mathrm{O} 3-\mathrm{C} 10-\mathrm{H} 4$ & 117.421 \\
\hline $\mathrm{C} 5-\mathrm{C} 6-\mathrm{C} 7$ & $122.5(6)$ & $\mathrm{C} 2-\mathrm{C} 10-\mathrm{H} 4$ & 117.409 \\
\hline $\mathrm{C} 6-\mathrm{C} 7-\mathrm{C} 9$ & $117.7(6)$ & & \\
\hline $\mathrm{C} 1-\mathrm{O} 1-\mathrm{C} 9-\mathrm{C} 7$ & $-179.9(4)$ & $\mathrm{C} 8-\mathrm{C} 4-\mathrm{C} 5-\mathrm{Br} 1$ & $-177.2(5)$ \\
\hline $\mathrm{C} 1-\mathrm{O} 1-\mathrm{C} 9-\mathrm{C} 8$ & $2.9(8)$ & $\mathrm{C} 8-\mathrm{C} 4-\mathrm{C} 5-\mathrm{C} 6$ & $1.4(9)$ \\
\hline $\mathrm{C} 9-\mathrm{O} 1-\mathrm{C} 1-\mathrm{C} 2$ & $-2.7(8)$ & $\mathrm{H} 2-\mathrm{C} 4-\mathrm{C} 5-\mathrm{Br} 1$ & 2.8 \\
\hline $\mathrm{C} 9-\mathrm{O} 1-\mathrm{C} 1-\mathrm{H} 1$ & 177.3 & $\mathrm{H} 2-\mathrm{C} 4-\mathrm{C} 5-\mathrm{C} 6$ & -178.6 \\
\hline $\mathrm{O} 1-\mathrm{C} 1-\mathrm{C} 2-\mathrm{C} 3$ & $2.5(8)$ & $\mathrm{H} 2-\mathrm{C} 4-\mathrm{C} 8-\mathrm{C} 3$ & -0.4 \\
\hline $\mathrm{O} 1-\mathrm{C} 1-\mathrm{C} 2-\mathrm{C} 10$ & $-179.8(5)$ & $\mathrm{H} 2-\mathrm{C} 4-\mathrm{C} 8-\mathrm{C} 9$ & 177.8 \\
\hline $\mathrm{H} 1-\mathrm{C} 1-\mathrm{C} 2-\mathrm{C} 3$ & -177.5 & $\mathrm{Br} 1-\mathrm{C} 5-\mathrm{C} 6-\mathrm{F} 1$ & $0.2(8)$ \\
\hline $\mathrm{H} 1-\mathrm{C} 1-\mathrm{C} 2-\mathrm{C} 10$ & 0.2 & $\mathrm{Br} 1-\mathrm{C} 5-\mathrm{C} 6-\mathrm{C} 7$ & $178.4(4)$ \\
\hline $\mathrm{C} 1-\mathrm{C} 2-\mathrm{C} 3-\mathrm{O} 2$ & $179.2(5)$ & $\mathrm{C} 4-\mathrm{C} 5-\mathrm{C} 6-\mathrm{F} 1$ & $-178.4(5)$ \\
\hline $\mathrm{C} 1-\mathrm{C} 2-\mathrm{C} 3-\mathrm{C} 8$ & $-2.2(8)$ & $\mathrm{C} 4-\mathrm{C} 5-\mathrm{C} 6-\mathrm{C} 7$ & $-0.2(9)$ \\
\hline $\mathrm{C} 1-\mathrm{C} 2-\mathrm{C} 10-\mathrm{O} 3$ & $0.3(9)$ & $\mathrm{F} 1-\mathrm{C} 6-\mathrm{C} 7-\mathrm{C} 9$ & $178.1(5)$ \\
\hline $\mathrm{C} 1-\mathrm{C} 2-\mathrm{C} 10-\mathrm{H} 4$ & -179.7 & $\mathrm{~F} 1-\mathrm{C} 6-\mathrm{C} 7-\mathrm{H} 3$ & -1.9 \\
\hline $\mathrm{C} 3-\mathrm{C} 2-\mathrm{C} 10-\mathrm{O} 3$ & $178.1(5)$ & $\mathrm{C} 5-\mathrm{C} 6-\mathrm{C} 7-\mathrm{C} 9$ & $-0.1(9)$ \\
\hline $\mathrm{C} 3-\mathrm{C} 2-\mathrm{C} 10-\mathrm{H} 4$ & -1.9 & $\mathrm{C} 5-\mathrm{C} 6-\mathrm{C} 7-\mathrm{H} 3$ & 179.9 \\
\hline $\mathrm{C} 10-\mathrm{C} 2-\mathrm{C} 3-\mathrm{O} 2$ & $1.4(8)$ & $\mathrm{C} 6-\mathrm{C} 7-\mathrm{C} 9-\mathrm{O} 1$ & $-177.9(5)$ \\
\hline $\mathrm{C} 10-\mathrm{C} 2-\mathrm{C} 3-\mathrm{C} 8$ & $-180.0(5)$ & $\mathrm{C} 6-\mathrm{C} 7-\mathrm{C} 9-\mathrm{C} 8$ & $-0.7(8)$ \\
\hline $\mathrm{O} 2-\mathrm{C} 3-\mathrm{C} 8-\mathrm{C} 4$ & $-0.8(8)$ & $\mathrm{H} 3-\mathrm{C} 7-\mathrm{C} 9-\mathrm{O} 1$ & 2.1 \\
\hline $\mathrm{O} 2-\mathrm{C} 3-\mathrm{C} 8-\mathrm{C} 9$ & $-178.9(5)$ & $\mathrm{H} 3-\mathrm{C} 7-\mathrm{C} 9-\mathrm{C} 8$ & 179.3 \\
\hline $\mathrm{C} 2-\mathrm{C} 3-\mathrm{C} 8-\mathrm{C} 4$ & $-179.4(5)$ & $\mathrm{C} 3-\mathrm{C} 8-\mathrm{C} 9-\mathrm{O} 1$ & $-2.9(8)$ \\
\hline $\mathrm{C} 2-\mathrm{C} 3-\mathrm{C} 8-\mathrm{C} 9$ & $2.4(7)$ & $\mathrm{C} 3-\mathrm{C} 8-\mathrm{C} 9-\mathrm{C} 7$ & $-179.9(5)$ \\
\hline $\mathrm{C} 5-\mathrm{C} 4-\mathrm{C} 8-\mathrm{C} 3$ & $179.6(5)$ & $\mathrm{C} 4-\mathrm{C} 8-\mathrm{C} 9-\mathrm{O} 1$ & $178.9(5)$ \\
\hline $\mathrm{C} 5-\mathrm{C} 4-\mathrm{C} 8-\mathrm{C} 9$ & $-2.2(8)$ & $\mathrm{C} 4-\mathrm{C} 8-\mathrm{C} 9-\mathrm{C} 7$ & $1.9(8)$ \\
\hline
\end{tabular}

Symmetry codes: (i) $x-1, y, z$; (ii) $x-1 / 2,-y+1 / 2,-z+3$; (iii) $x+1, y, z$; (iv) $x+1 / 2,-y+1 / 2,-z+3$; (v) $x, y, z+1$; (vi) $x+1, y, z+1$; (vii) $-x+3 / 2,-y, z+1 / 2$; (viii) $x-1, y, z-1$; (ix) $x, y, z-1$; (x) $-x+3 / 2,-y, z-1 / 2$; (xi) $-x+1 / 2,-y, z-1 / 2$; (xii) $-x+1 / 2,-y, z+1 / 2$. 


\section{supporting information}

Hydrogen-bond geometry $\left(\AA,{ }^{\circ}\right)$

\begin{tabular}{lllll}
\hline$D-\mathrm{H}^{\cdots} A$ & $D-\mathrm{H}$ & $\mathrm{H} \cdots A$ & $D \cdots A$ & $D-\mathrm{H} \cdots A$ \\
\hline $\mathrm{C} 1-\mathrm{H} 1 \cdots \mathrm{O}^{\text {vii }}$ & 0.95 & 2.41 & $3.240(7)$ & 146 \\
$\mathrm{C} 7-\mathrm{H} 3 \cdots 2^{\text {vi }}$ & 0.95 & 2.26 & $3.166(7)$ & 158 \\
\hline
\end{tabular}

Symmetry codes: (vi) $x+1, y, z+1$; (vii) $-x+3 / 2,-y, z+1 / 2$. 